\title{
Allosteric Activation of Cytochrome P450 3A4 via Progesterone Bioconjugation
}

\author{
Vanja Polic, and Karine Auclair* \\ Department of Chemistry, McGill University, 801 Sherbrooke Street West, Montreal, Quebec, Canada H3A 0B8
}

\begin{abstract}
Human cytochrome P450 3A4 (CYP3A4) is responsible for the metabolism of the majority of drugs. As such, it is implicated in many adverse drug-drug and food-drug interactions, and is of significant interest to the pharmaceutical industry. This enzyme is known to simultaneously bind multiple ligands and display atypical enzyme kinetics, suggestive of allostery and cooperativity. As well, evidence of a postulated peripheral allosteric binding site has provoked debate around its significance and location. We report the use of bioconjugation to study the significance of substrate binding at the proposed allosteric site and its effect on CYP3A4 activity. CYP3A4 mutants were created and covalently modified with various small molecules including progesterone. The labeled mutants displayed enhanced kinetic stability and improved activity in testosterone and 7-benzyloxy-(4-trifluoromethyl)coumarin oxidation assays. Our work applies a new strategy to study cytochrome P450 allostery and supports the hypothesis that substrate binding at the postulated allosteric site of CYP3A4 may induce functional cooperativity.
\end{abstract}

Cytochrome P450 enzymes (P450s) are a large family of heme-dependent monooxygenase enzymes found throughout all domains of life. They are involved in the biosynthesis of a large variety of molecules such as steroid hormones, cofactors, and antibiotics, as well as in the metabolism of xenobiotics. ${ }^{1}$ Human cytochrome P450 3A4 (CYP3A4) is responsible for the metabolism of over $50 \%$ of existing drugs and is implicated in many drugdrug, food-drug and natural health product-drug interactions. ${ }^{1,2}$

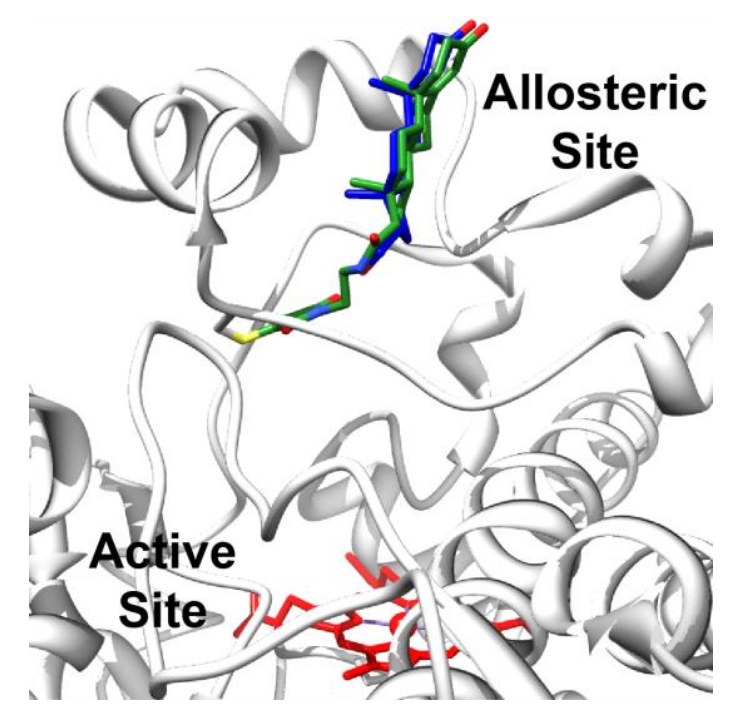

Figure 1. A modified X-ray crystal structure image showing progesteronemaleimide (Pg-M) conjugated to Cys215. Pg-M is shown in green superimposed on the existing, co-crystallized progesterone in blue. Pg-M was built into the allosteric site using the USCF Chimera package ${ }^{3}$, PDB structure $1 \mathrm{~W}^{\mathrm{F}} \mathrm{F}^{4}$ and the Persistence of Vision TM Raytracer.
CYP3A4 has a large, flexible active site allowing it to bind substrates varying greatly in size, or to bind multiple smaller substrates simultaneously. In the case of testosterone, it is thought that CYP3A4 can bind up to 3 molecules concurrently, at least two of which may bind at the active site, as suggested by spectroscopic binding studies. ${ }^{5}$ The ability of CYP3A4 to bind multiple substrates at once may explain, at least in part, the atypical kinetics observed for some of its reactions, as evident in Michaelis-Menten plots. ${ }^{6-9}$

CYP3A4 can also show homotropic and heterotropic cooperativity, a phenomenon with clinical importance due to its implication in drug metabolism and drug interactions. ${ }^{2,10}$ Substrate binding to CYP3A4 is typically spectrally observed due to associated changes in the heme spin state. Since for most ligands the first binding event cannot be observed spectroscopically, it is considered "spin state silent", and away from the heme. The presence of true functional cooperativity is therefore debated and may be the result of a "silent" artifact. ${ }^{5}$ Ligand interactions with CYP3A4 4 , 10-27 remain challenging to investigate and new approaches are required.

An X-ray crystallography structure of CYP3A4 showing a co-crystallized progesterone molecule in a region far from the heme reactive center has alluded to the possibility of a peripheral binding site responsible for allosteric effects (Figure 1). ${ }^{4}{ }^{28}$ However, the significance of the co-crystallized progesterone and the peripheral site remain contentious due to progesterone binding at the interface of a non-naturally existing protein dimer. ${ }^{28}$ Still, residues in this region have also been implicated in CYP3A4 cooperativity via site-directed mutagenesis. ${ }^{19,21}$, ${ }^{22}$ As well, Förster resonance energy transfer studies have shown that binding of a substrate in this region may be involved in a ligand-induced allosteric transition. ${ }^{29}$ 
The work presented herein demonstrates the utility of site-selective bioconjugation to confirm the relevance of the allosteric binding pocket to CYP3A4 activity. This approach has previously been used to locate new, and study the effects of existing, allosteric sites on select enzymes, but has never before been applied to a P450 enzyme. ${ }^{30-36}$ A progesterone analogue was covalently attached to the postulated allosteric site in different orientations (Figure 1) and the modified enzyme was studied for its activity towards 7-benzyloxy-(4trifluoromethyl)coumarin (BFC) and testosterone. We report here the modulation of CYP3A4 activity in the presence of progesterone covalently bound to the allosteric site and discuss the significance of these findings.

Our first attempt at bioconjugation to CYP3A4 involved the use of unnatural amino acid $o$-propargyl tyrosine and the copper catalyzed azide-alkyne cyclization reaction, never before reported with CYP3A4. Unfortunately the reaction conditions proved to be harmful to the enzyme (see section S6). We next used cysteine mutants combined with maleimide derivatives based on previous success reported for this bioconjugation strategy. ${ }^{37,38} \mathrm{~A}$ cysteine-depleted mutant (Mutant 1) was generated as the starting point for a series of six mutants in which selected allosteric site amino acids were separately replaced with a cysteine (L211C,

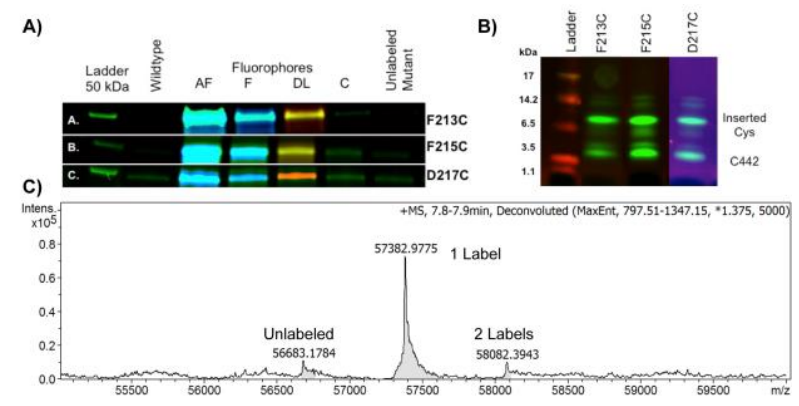

Figure 2. Fluorophore bioconjugation using cysteine-maleimide chemistry. A) SDS-PAGE segments showing bands corresponding to CYP3A4. Fluorophores are abbreviated: AF - AlexaFluor 488, F - Fluorescein, DL DyLight550 and C - Coumarin. Blue bands correspond to AlexaFluor 488 and fluorescein, orange/yellow bands to DyLight550, green bands to unlabeled proteins or proteins labeled with coumarin. B) Sample Tricine-SDS-PAGE results for bioconjugation of AlexaFluor 488 to each $\mathrm{CNBr}$ digested CYP3A4 mutant. Green bands are due to peptides labeled with the fluorophore, while red bands correspond to the unlabeled protein ladder. See supplemental information for remaining fluorophores (Figures S7-S9) C) Sample deconvoluted HPLC-MS-QToF spectrum showing results for mutant D217C labeled by AlexaFluor 488. See supplemental information for remaining fluorophores (Section S4)

F213C, D214C, F215C, D217C, and V240C, Table S1). Mutant 1 included the mutation of all accessible cysteines, with only 2 native cysteines remaining, the buried $\mathrm{C} 377^{37}$ and the proximal heme iron ligand $\mathrm{C} 442$. Three of the six allosteric site CYP3A4 cysteine mutants oxidized testosterone with total turnovers about twice as high as that of the wildtype enzyme suggesting higher stability, and only mutant 1 and mutant F213C showed improved initial rates of BFC transformation (Figure $\mathrm{S} 1$ ).

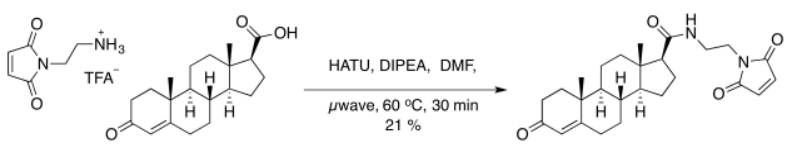

Scheme 1. Synthesis of maleimide-containing progesterone derivative Pg-M.

In order to rapidly establish the synthetic methodology, mutants F213C, F215C and D217C were reacted with the maleimide derivatives of four different fluorophores: AlexaFluor 488, fluorescein, DyLight 550 and N-7dimethylamino-4-methylcoumarin (abbreviated as coumarin). All four fluorophores were successfully conjugated as confirmed by SDS-PAGE (Figure 2A, S2S4) and the reaction yield was determined using HPLCMS-QToF (Figure 2C and S5) In all cases, the mutants were primarily labeled with only one fluorophore molecule, with the presence of little unlabeled or doubly labeled enzyme. Di-labeled enzyme likely resulted from the reaction at $\mathrm{C} 442$ of the denatured enzyme, as suggested by the SDS-PAGE of the cyanogen bromidedigested protein (vide infra). The yield of mono-labeled protein ranged from $45-88 \%$, and the degree of labeling seemed to improve slightly with smaller, less charged and more aromatic molecules. Control reactions where the maleimide moiety of the fluorophores was quenched using dithiothreitol (DTT) before reaction with the cysteine mutants suggest that conjugation does not occur in absence of a functional maleimide moiety and that the free fluorophores are fully washed out in the process (Figure S6).

To verify the regioselectivity of the bioconjugation, the enzyme samples were subjected to digestion using cyanogen bromide, followed by analysis of the peptides using tricine-SDS-PAGE, as previously established by our group. ${ }^{37}$ All results are consistent with the introduced cysteine being the major site of bioconjugation, with a smaller portion of labeling at the heme proximal cysteine (C442) (Figures 1B and S7-S9). Labeling at C442 implies that some enzyme was denatured during the conjugation reaction, which is to be expected for CYP3A4 due to its low stability. The characterization above suggests this denatured protein species to be a minor component, which should not interfere with the analysis of enzyme activity since it is inactivated and likely aggregates and precipitates out of solution.

The effects of the conjugated fluorophores on CYP3A4 turnover and initial rates of reaction were studied with the substrates testosterone and BFC respectively. It was clear that all labeled mutants performed comparably to the unlabeled controls for the testosterone assay, except for the coumarin conjugate of 
the F213C mutant, which showed improved turnover (Figure S10A). This agrees with previous reports showing
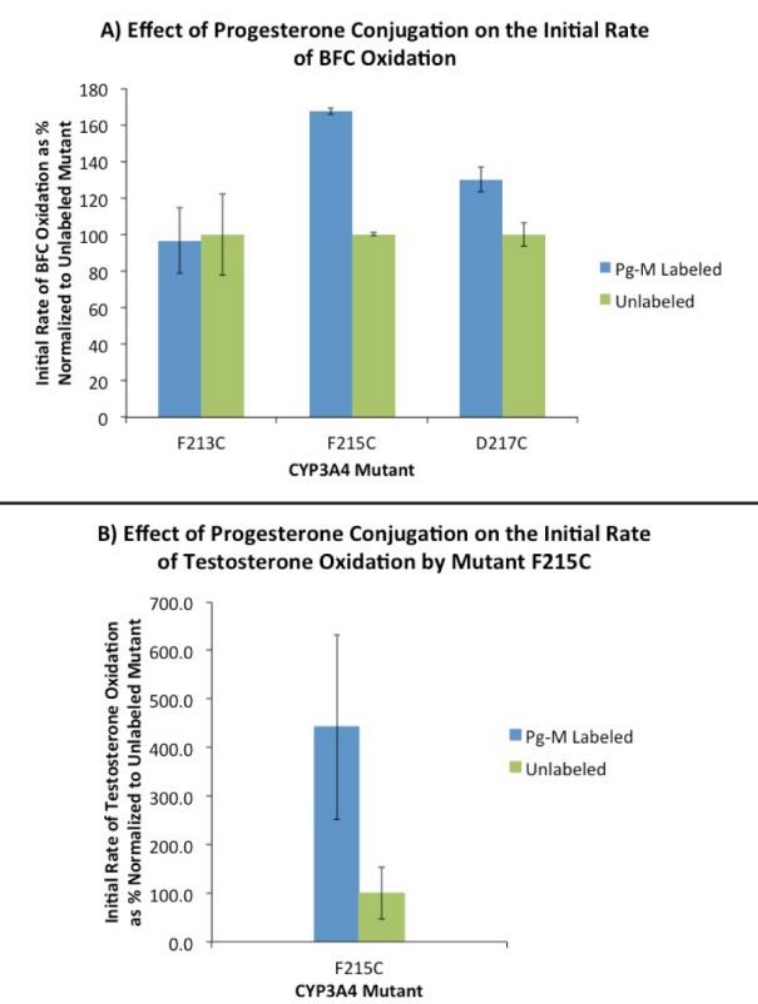

Figure 3. Effect of conjugated Pg-M on the initial rate of CYP3A4 mutants. A) Effect of conjugated Pg-M on BFC transformation by CYP3A4 mutants. Activity data for each mutant compares the initial rate of BFC transformation and is normalized to the initial rate of the corresponding unlabeled mutant. The reaction consisted of $0.4 \mu \mathrm{M} \mathrm{CYP} 3 \mathrm{~A} 4,0.8 \mu \mathrm{M} \mathrm{CPR}, 33 \mu \mathrm{M} \mathrm{BFC}$ and 1.1 $\mathrm{mM}$ NADPH in a $100 \mu \mathrm{L}$ volume. Each data point is the average of three experiments with standard error shown with error bars. B) Effect of conjugated Pg-M on testosterone hydroxylation by CYP3A4 mutants. Comparison of the initial rate of testosterone transformation by the bioconjugated mutant normalized to the initial rate of the corresponding unlabelled mutant. The reaction consisted of $0.8 \mu \mathrm{M}$ CYP3A4, $0.8 \mu \mathrm{M}$ CPR, $80 \mu \mathrm{M}$ testosterone and $1 \mathrm{mM}$ NADPH in a $100 \mu \mathrm{L}$ volume. Each data point is the average of three experiments with standard deviation shown with error bars.

that coumarin derivatives can have a stimulating effect on testosterone metabolism by CYP3A4. ${ }^{15,19}$ It was expected that the orientation of the coumarin in the pocket would matter, and this is supported by the absence of a positive effect on the enzyme rate observed for the $\mathrm{F} 215 \mathrm{C}$ and $\mathrm{D} 217 \mathrm{C}$ mutants. Interestingly, the rate of $\mathrm{BFC}$ transformation to HFC was reduced from $20-60 \%$ with bioconjugation for all but the coumarin bioconjugates (Figure S10B). A slight increase in initial rate was observed for the coumarin-labeled CYP3A4, however for mutants F215C instead of F213C. This supports previous reports that CYP3A4 allostery may be substrate- and effector-dependent. ${ }^{15,39}$
Due to its implication in both homotropic and heterotropic cooperativity, we were interested in studying the effect of a known CYP3A4 substrate and postulated

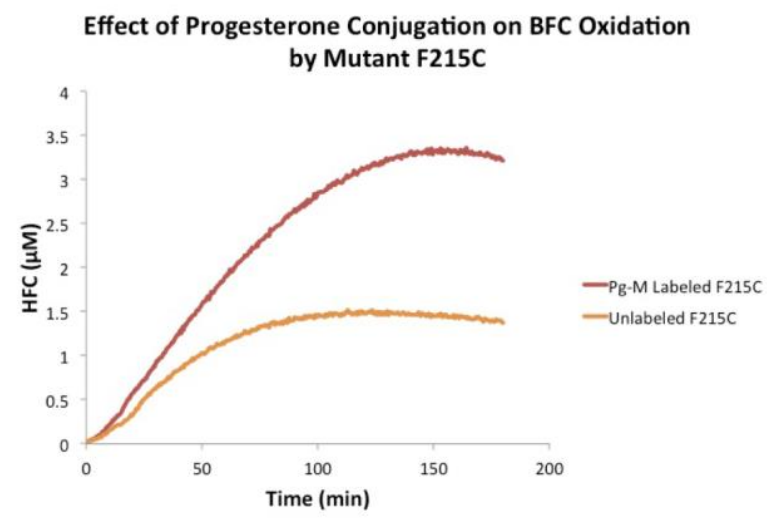

Figure 4. Effect of conjugated Pg-M on the kinetic stability of Mutant F215C. The enzyme activity was measured using the BFC assay. All samples were exposed to bioconjugation conditions as detailed in Section 2.6 of the Supporting Information. The reaction consisted of $0.4 \mu \mathrm{M}$ CYP3A4, $0.8 \mu \mathrm{M}$ $\mathrm{CPR}, 33 \mu \mathrm{M} \mathrm{BFC}$ and $10 \mathrm{mM} \mathrm{NADPH}$ in a $100 \mu \mathrm{L}$ volume. Results are shown as the average of three experiments.

allosteric site ligand, progesterone, on enzyme activity. Using manual fitting, an artificially conjugated maleimide containing progesterone analogue Pg-M with a 2 carbon linker allowed for superimposition of the progesterone moiety on the existing co-crystallized progesterone (Figure 1). Thus, Pg-M was rationally designed and synthesized starting from commercially available $17 \beta-$ testosterone carboxylic acid (Scheme 1). Using the same reaction conditions as used for the fluorophores, Pg-M was conjugated to each of the three mutants. Each mutant was primarily mono-labeled $(>80 \%)$ with small amounts of unlabeled $(<10 \%)$ and doubly-labeled $(<15 \%)$ species observed (Figure S11). After conjugation and extensive removal of excess Pg-M, some enzyme was found in the high spin state (estimated to $<25 \%$ ). This may be due to the high flexibility of CYP3A4 and the partial translocation of conjugated $\mathrm{Pg}-\mathrm{M}$ from the allosteric site to the active site. The effect of progesterone conjugation on CYP3A4 was investigated and overall the presence of progesterone had a positive effect on the initial rate of substrate oxidation by CYP3A4 towards both testosterone and BFC (Figure 3). This was seen for two out of the three mutants, but was most pronounced for mutant F215C. These results support our hypothesis that progesterone conjugation may mimic the allosteric effect that it has in the transformation of other substrates, whether through allosteric site binding or partial reorientation in the active site $(<25 \%) .6,10,16,19,21,22,40-48$ As expected, the orientation of the molecule in the pocket (as simulated here using different mutants) influences allostery. 
Since the F215C mutation resulted in a decrease in enzyme activity relative to the parent mutant 1 (especially with BFC, see Figure S1) we investigated whether the conjugation of Pg-M was compensating for the removal of F215. This was achieved by conjugating the smaller $N$ methylmaleimide (MM) as a Phe mimic. The F215C-MM

Table 1. Kinetic parameters for BFC and testosterone transformation by mutant F215C

\begin{tabular}{|c|c|c|c|}
\hline \multirow{2}{*}{ Sample } & \multicolumn{2}{|c|}{ BFC } & \multirow{2}{*}{$\begin{array}{c}\text { TST } \\
k_{\text {cat }}\left(\min ^{-1}\right)\end{array}$} \\
\hline & $K_{\mathrm{M}}(\mu \mathrm{M})$ & $k_{\text {cat }}\left(\min ^{-1}\right)$ & \\
\hline Wildtype CYP3A4 & $1.2 \pm 0.5$ & $\begin{array}{r}0.0011 \pm \\
0.0001\end{array}$ & $0.5 \pm 0.2$ \\
\hline F215C-Unlabeled & $9 \pm 4$ & $\begin{array}{r}0.0036 \pm \\
0.0005\end{array}$ & $0.11 \pm 0.04$ \\
\hline F215C-PgM & $27 \pm 7$ & $0.11 \pm 0.01$ & $0.45 \pm 0.06$ \\
\hline
\end{tabular}

conjugate displayed initial rate of $\mathrm{BFC}$ transformation that was significantly less than that of the F215C-Pg-M conjugate (Figure S12), suggesting that $\mathrm{Pg}-\mathrm{M}$ is more than just compensating for the removed Phe residue. Furthermore, Michaelis-Menten analysis of mutant F215C for BFC transformation, before and after bioconjugation to Pg-M, showed marked differences in the kinetic parameters (Table 1 and Figure S13). The $k_{\text {cat }}$ for the reaction with BFC was greater for the Pg-M labeled mutant, implying that conjugation of progesterone improves the initial rates of BFC transformation. The increase in $K_{\mathrm{M}}$ also aligns with the assumption that the multiple binding events occur with differing binding affinities. ${ }^{40}$ Our results suggest that the first binding event may bind with higher affinity (as proposed by others), ${ }^{40}$ and once blocked due to conjugation, the apparent $\mathrm{K}_{M}$ is expected to increase. Conjugation of progesterone also improves the initial rates of testosterone hydroxylation. Due to issues with substrate precipitation in buffer, ${ }^{49}$ low enzyme activity and protein aggregation, ${ }^{50,51}$ we were unable to obtain reliable $K_{\mathrm{M}}$ for testosterone (Figure S14).

The positive effect of progesterone was also evident when studying the kinetic stability of mutant F215C during BFC transformation (Figure 4). Conjugation of progesterone at this site not only improved the initial rate of BFC transformation, but it also stabilized the enzyme during catalysis such that the activity plateaued at a later time. These results suggest for the first time that progesterone binding at an allosteric pocket may also have a stabilizing effect on CYP3A4 in addition to improving its activity.

In summary, we have demonstrated how substrate bioconjugation can be used to investigate the presence of a proposed allosteric binding site. To our knowledge, this methodology has not been applied to study the complex behavior of P450 enzymes. Interestingly, the strategic attachment of a coumarin derivative or of progesterone to CYP3A4 proved to be a successful strategy to reproduce the allosteric effect observed with these ligands. As such, the bioconjugates reported herein, and possibly other variants, ought to greatly simplify further studies of the complex cooperative and allosteric behavior of CYP3A4.

In light of the role of CYP3A4 in human metabolism, the bioconjugates reported here can also find use in the study of drug metabolism and drug interactions. CYP3A4 is not only the major actor in drug metabolism but also an unequalled catalyst, made so by its ability to bind a large variety of ligands and selectively catalyze many reactions, such as hydroxylations at unactivated $\mathrm{C}-\mathrm{H}$ bonds, a reaction that remains mostly inaccessible to chemical catalysis. The enzyme activation and improved kinetic stability observed with the bioconjugates may be harnessed to realize new biocatalytic applications of this enzyme.

\section{ASSOCIATED INFORMATION}

\section{Supporting Information}

The Supporting Information is available free of charge on the ACS Publications website.

Syntheses and characterization of the unnatural amino acid $O$-propargyl tyrosine $(\mathrm{OPgY})$, mutagenesis primer sequences, full details for each mutant and conjugation reactions regarding SDS-PAGE, HPLC-MS-QToF, supplemental figures described in text (PDF)

\section{AUTHOR INFORMATION}

\section{Corresponding Author}

* karine.auclair@mail.mcgill.ca

\section{ACKNOWLEDGEMENTS}

This research was funded by the National Science and Engineering Research Council of Canada (NSERC) and the Center in Green Chemistry and Catalysis. V.P. was supported by scholarships from the Dr. Richard H. Tomlinson Foundation, Walter C. Sumner Foundation, the Centre in Green Chemistry and Catalysis, and NSERC. We would also like to thank Dr. J. R. Halpert for providing the CYP3A4 plasmid, Dr. F. P. Guengerich for the CPR plasmid, Dr. P. G. Schultz for the unnatural aminoacyl-tRNA synthetase/tRNA pairs plasmid, Dr. A. S. Wahba for his work on protein LC-MS-QToF, Dr. I. Sevrioukova for helpful discussions and Genome Quebec for DNA sequencing. Molecular graphics and analyses were performed with the UCSF Chimera package. Chimera is developed by the Resource for Biocomputing, Visualization, and Informatics at the University of California, San Francisco (supported by NIGMS P41-GM103311).

\section{REFERENCES}


(1) Ortiz de Montellano, P. R. (2015) Cytochrome P450: Structure, mechanism, and biochemistry, 4 ed., Springer International Publishing, New York.

(2) Guengerich, F. P. (1999) Cytochrome P-450 3A4: regulation and role in drug metabolism. Annu. Rev. Pharmacol. Toxicol. 39, 1-17.

(3) Pettersen, E. F., Goddard, T. D., Huang, C. C., Couch, G. S., Greenblatt, D. M., Meng, E. C., and Ferrin, T. E. (2004) UCSF Chimera--a visualization system for exploratory research and analysis. J. Comput. Chem. 25, 1605-1612.

(4) Williams, P. A., Cosme, J., Vinkovic, D. M., Ward, A., Angove, H. C., Day, P. J., Vonrhein, C., Tickle, I. J., and Jhoti, H. (2004) Crystal structures of human cytochrome P450 3A4 bound to metyrapone and progesterone. Science 305, 683-686.

(5) Denisov, I. G., Baas, B. J., Grinkova, Y. V., and Sligar, S. G. (2007) Cooperativity in cytochrome P450 3A4: linkages in substrate binding, spin state, uncoupling, and product formation. J. Biol. Chem. 282, 7066-7076.

(6) Houston, J. B., and Kenworthy, K. E. (2000) In vitro-in vivo scaling of cyp kinetic data not consistent with the classical Michaelis-Menten model. Drug Metab. Dispos. 28, 246-254.

(7) Hutzler, J. M., and Tracy, T. S. (2002) Atypical kinetic profiles in drug metabolism reactions. Drug Metab. Dispos. 30, 355-362.

(8) Shou, M., Mei, Q., Ettore, J., Michael W, Dai, R., Baille, T. A., and Rushmore, T. H. (1999) Sigmoidal kinetic model for two co-operative substrate-binding sites in a cytochrome P450 3A4 active site: an example of the metabolism of diazepam and its derivatives. Biochem. J 340, 845.

(9) Ueng, Y. F., Kuwabara, T., Chun, Y. J., and Guengerich, F. P. (1997) Cooperativity in oxidations catalyzed by cytochrome P450 3A4. Biochemistry 36, 370381.

(10) Denisov, I. G., Frank, D. J., and Sligar, S. G. (2009) Cooperative properties of cytochromes P450. Pharmacol. Ther. 124, 151-167.

(11) Atkins, W. M. (2004) Implications of the allosteric kinetics of cytochrome P450s. Drug Discovery Today 9, 478484.

(12) Atkins, W. M. (2005) Non-Michaelis-Menten Kinetics in Cytochrome P450-Catalyzed Reactions. Annu. Rev. Pharmacol. Toxicol. 45, 291-310.

(13) Davydov, D. R., Baas, B. J., Sligar, S. G., and Halpert, J. R. (2007) Allosteric mechanisms in cytochrome P450 3A4 studied by high-pressure spectroscopy: Pivotal role of substrate-induced changes in the accessibility and degree of hydration of the heme pocket. Biochemistry 46, 7852-7864.

(14) Davydov, D. R., Davydova, N. Y., Sineva, E. V., Kufareva, I., and Halpert, J. R. (2013) Pivotal role of P450P450 interactions in CYP3A4 allostery: the case of alphanaphthoflavone. Biochem. J 453, 219-230.

(15) Davydov, D. R., Davydova, N. Y., Tsalkova, T. N., and Halpert, J. R. (2008) Effect of glutathione on homo- and heterotropic copperativity in cytochrome P450 3A4. Arch. Biochem. Biophys. 471, 134-145.

(16) Davydov, D. R., and Halpert, J. R. (2008) Allosteric P450 mechanisms: multiple binding sites, multiple conformers or both? Expert Opin. Drug Metab. Toxicol. 4, 1523-1535.

(17) Denisov, I. G., Mak, P. J., Grinkova, Y. V., Bastien, D., Bérubé, G., Sligar, S. G., and Kincaid, J. R. (2016) The use of isomeric testosterone dimers to explore allosteric effects in substrate binding to cytochrome P450 CYP3A4. J. Inorg. Biochem. 158, 77-85.

(18) Denisov, I. G., and Sligar, S. G. (2012) A novel type of allosteric regulation: Functional cooperativity in monomeric proteins. Arch. Biochem. Biophys. 519, 91-102.

(19) Domanski, T. L., He, Y.-A., Khan, K. K., Roussel, F., Wang, Q., and Halpert, J. R. (2001) Phenylalanine and tryptophan scanning mutagenesis of CYP3A4 substrate recognition site residues and effect on substrate oxidation and cooperativity. Biochemistry 40, 10150-10160.

(20) Frank, D. J., Denisov, I. G., and Sligar, S. G. (2009) Mixing apples and oranges: Analysis of heterotropic cooperativity in cytochrome P450 3A4. Arch. Biochem. Biophys. 488, 146-152.

(21) Harlow, G. R., and Halpert, J. R. (1997) Alaninescanning mutagenesis of a putative substrate recognition site in human cytochrome P450 3A4. J. Biol. Chem. 272, 53965402.

(22) Harlow, G. R., and Halpert, J. R. (1998) Analysis of human cytochrome P450 3A4 cooperativity: construction and characterization of a site-directed mutant that displays hyperbolic steroid hydroxylation kinetics. Proc. Natl. Acad. Sci. U.S. A. 95, 6636-6641.

(23) Müller, C. S., Knehans, T., Davydov, D. R., Bounds, P. L., von Mandach, U., Halpert, J. R., Caflisch, A., and Koppenol, W. H. (2015) Concurrent Cooperativity and Substrate Inhibition in the Epoxidation of Carbamazepine by Cytochrome P450 3A4 Active Site Mutants Inspired by Molecular Dynamics Simulations. Biochemistry 54, 711-721.

(24) Roberts, A. G., Yang, J., Halpert, J. R., Nelson, S. D., Thummel, K. T., and Atkins, W. M. (2011) The structural basis for homotropic and heterotropic cooperativity of midazolam metabolism by human cytochrome P450 3A4. Biochemistry 50, 10804-10818.

(25) Sligar, S. G., and Denisov, I. G. (2007) Understanding Cooperativity in Human P450 Mediated Drug-Drug Interactions. Drug Metab. Rev. 39, 567-579.

(26) Woods, C. M., Fernandez, C., Kunze, K. L., and Atkins, W. M. (2011) Allosteric activation of cytochrome P450 3A4 by $\alpha$-naphthoflavone: Branch point regulation revealed by isotope dilution analysis. Biochemistry 50, 10041-10051.

(27) Yang, J., Atkins, W. M., Isoherranen, N., Paine, M. F., and Thummel, K. E. (2012) Evidence of CYP3A allosterism in vivo: analysis of interaction between fluconazole and midazolam. Clin. Pharmacol. Ther. 91, 442-449.

(28) Sevrioukova, I. F., and Poulos, T. L. (2015) AnionDependent Stimulation of CYP3A4 Monooxygenase. Biochemistry 54, 4083-4096.

(29) Davydov, D. R., Rumfeldt, J. A. O., Sineva, E. V., Fernando, H., Davydova, N. Y., and Halpert, J. R. (2012) Peripheral ligand-binding site in cytochrome P450 3A4 located with fluorescence resonance energy transfer (FRET). J. Biol. Chem. 287, 6797-809.

(30) Davie, B. J., Sexton, P. M., Capuano, B., Christopoulos, A., and Scammells, P. J. (2014) Development of a Photoactivatable Allosteric Ligand for the $M 1$ 
Muscarinic Acetylcholine Receptor. ACS Chem. Neurosci. 5, 902-907.

(31) Gregory, K. J., Velagaleti, R., Thal, D. M., Brady, R. M., Christopoulos, A., Conn, P. J., and Lapinsky, D. J. (2016) Clickable Photoaffinity Ligands for Metabotropic Glutamate Receptor 5 Based on Select Acetylenic Negative Allosteric Modulators. ACS Chem. Biol. 11, 1870-1879.

(32) Hardy, J. A., Lam, J., Nguyen, J. T., O'Brien, T., and Wells, J. A. (2004) Discovery of an allosteric site in the caspases. Proc. Natl. Acad. Sci. U. S. A. 101, 12461-12466.

(33) Hegazy, U. M., Musdal, Y., and Mannervik, B. (2013) Hidden allostery in human glutathione transferase P1-1 unveiled by unnatural amino acid substitutions and inhibition studies. J. Mol. Biol. 425, 1509-1514.

(34) Sadowsky, J. D., Burlingame, M. A., Wolan, D. W., McClendon, C. L., Jacobson, M. P., and Wells, J. A. (2011) Turning a protein kinase on or off from a single allosteric site via disulfide trapping. Proc. Natl. Acad. Sci. U. S. A. 108, 6056-6061.

(35) Scheer, J. M., Romanowski, M. J., and Wells, J. A. (2006) A common allosteric site and mechanism in caspases. Proc. Natl. Acad. Sci. U. S. A. 103, 7595-7600.

(36) Warner, J. B., Muthusamy, A. K., and Petersson, E. J. (2014) Specific modulation of protein activity by using a bioorthogonal reaction. ChemBioChem 15, 2508-2514.

(37) Ménard, A., Huang, Y., Karam, P., Cosa, G., and Auclair, K. (2012) Site-Specific Fluorescent Labeling and Oriented Immobilization of a Triple Mutant of CYP3A4 via C64. Bioconjugate Chem. 23, 826-836.

(38) Tsalkova, T. N., Davydova, N. Y., Halpert, J. R., and Davydov, D. R. (2007) Mechanism of interactions of alphanaphthoflavone with cytochrome P450 3A4 explored with an engineered enzyme bearing a fluorescent probe. Biochemistry 46, 106-119.

(39) Sevrioukova, I. F., and Poulos, T. L. (2013) Understanding the mechanism of cytochrome P450 3A4: recent advances and remaining problems. Dalton Trans. 42, 3116-3126.

(40) Denisov, I. G., Grinkova, Y. V., Baylon, J. L., Tajkhorshid, E., and Sligar, S. G. (2015) Mechanism of Drug-Drug Interactions Mediated by Human Cytochrome P450 CYP3A4 Monomer. Biochemistry 54, 2227-2239.

(41) Domanski, T. L., He, Y. A., Harlow, G. R., and Halpert, J. R. (2000) Dual role of human cytochrome P450 3A4 residue Phe-304 in substrate specificity and cooperativity. J. Pharmacol. Exp. Ther. 293, 585-591.

(42) Domanski, T. L., Liu, J., Harlow, G. R., and Halpert, J. R. (1998) Analysis of four residues within substrate recognition site 4 of human cytochrome P450 3A4: role in steroid hydroxylase activity and alpha-naphthoflavone stimulation. Arch. Biochem. Biophys. 350, 223-232.

(43) Fernando, H., Davydov, D. R., Chin, C. C., and Halpert, J. R. (2007) Role of subunit interactions in P450 oligomers in the loss of homotropic cooperativity in the cytochrome P450 3A4 mutant L211F/D214E/F304W. Arch. Biochem. Biophys. 460, 129-140.

(44) He, Y.-A., He, Y. Q., Szklarz, G. D., and Halpert, J. R. (1997) Identification of three key residues in substrate recognition site 5 of human cytochrome P450 3A4 by cassette and site-directed mutagenesis. Biochemistry 36, 8831-8839.
(45) Henshall, J., Galetin, A., Harrison, A., and Houston, J. B. (2008) Comparative analysis of CYP3A heteroactivation by steroid hormones and flavonoids in different in vitro systems and potential in vivo implications. Drug Metab. Dispos. 36, 1332-1340.

(46) Khan, K. K., He, Y. Q., Domanski, T. L., and Halpert, J. R. (2002) Midazolam Oxidation by Cytochrome P450 3A4 and Active-Site Mutants: an Evaluation of Multiple Binding Sites and of the Metabolic Pathway That Leads to Enzyme Inactivation. Mol. Pharmacol. 61, 495-506.

(47) Niwa, T., Murayama, N., and Yamazaki, H. (2008) Heterotropic cooperativity in oxidation mediated by cytochrome p450. Curr. Drug Metab. 9, 453-462.

(48) Schwab, G. E., Raucy, J. L., and Johnson, E. F. (1988) Modulation of rabbit and human hepatic cytochrome P-450catalyzed steroid hydroxylations by alpha-naphthoflavone. Mol. Pharmacol. 33, 493-499.

(49) Isin, E. M., and Guengerich, F. P. (2006) Kinetics and Thermodynamics of Ligand Binding by Cytochrome P450 3A4. J. Biol. Chem. 281, 9127-9136.

(50) Das, A., Zhao, J., Schatz, G. C., Sligar, S. G., and Van Duyne, R. P. (2009) Screening of type I and II drug binding to human cytochrome P450-3A4 in nanodiscs by localized surface plasmon resonance spectroscopy. Anal. Chem. 81, 3754-3759.

(51) Trubetskoy, O. V., Gibson, J. R., and Marks, B. D. (2005) Highly miniaturized formats for in vitro drug metabolism assays using vivid fluorescent substrates and recombinant human cytochrome P450 enzymes. J. Biomol. Screen. 10, 56-66. 


\section{TABLE OF CONTENTS GRAPHIC}

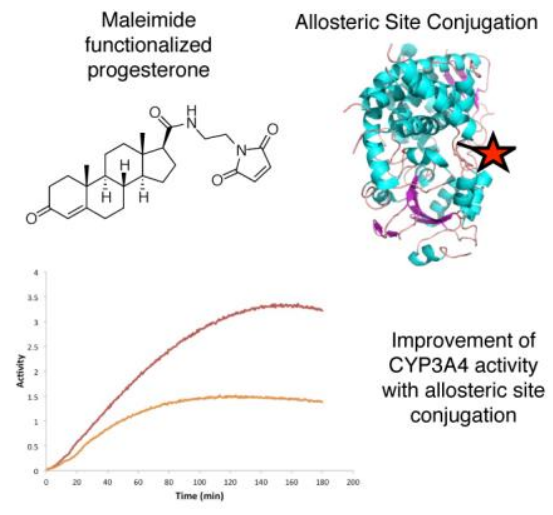

